\title{
Cuivre, foie et système nerveux
}

En 1912, un neurologiste anglais, S.A.Kínníer Wilson, décrivit sous le nom de «dégénérescence lenticulaire progressive» 4 observations d'une association nosologique nouvelle composée d'un syndrome extrapyramidal avec cirrhose hépatique. II imaginait une relation de 256

Editorial

cause à effet entre les deux atteintes organiques, et $\Gamma$ on parla alors de «cirrhoses nerveuses». Les travaux modernes ont rompu ce lien de causalité, en mettant en evidence des perturbations métaboliques, concernant principalement le cuivre, et qui seraient responsables des lesions hépatiques et nerveuses observées, simultanément ou séparé-ment.

En effet, si la dégénérescence hépato-lenticulaire (D.H.-L.) est composée d'une triade classique hypertonie extrapyramidale et tremblement; cirrhose hépatique; anneau cornéen (bande circulaire vert bronze) - il est habituel qu'un des deux systèmes perturbés s'exprime aux dépens de l'autre. Dans une remarquable monographic («Dégénérescence hêpato-lentículaíre»; 250 pp.; 46 fig., dont 7 en couleurs; Masson edit., Paris 1959), G. Boudín et B. Pépín vien-nent de peindre un tableau minutieux de nos connaissances sur cette curieuse maladie, où $\Gamma$ on trouvera tous les details nécessaires sur Vaspect neurologíque de la question; la cirrhose est alors latente, et Tattention peut être attirée sur le foie dans deux circonstances: il existe souvent des poussées ictériques successives au stade pré-neurologique; la mort est parfois due à une complication hépatique, par hémorragie digestive notamment. Chose curieuse, si la cirrhose est en general de forme atrophique à grosses nodosités, les tests fonc-tionnels dépassent rarement les limites de la normale, et il faut faire appel à la ponction-biopsie pour avoir une preuve de la sclérose hépatique.

Les formes abdomínales de la D.H.-L. représentent seulement le quart des cas connus, et frappent surtout les jeunes; quand $\Gamma$ affec-tion évolue de façon subaiguë, on parle même d'«Abdominal Wilson». La forme ascitique pure simule la tuberculose péritonéale; la forme hépato-splénomégalique realise le tableau d'une cirrhose juvenile et s'accompagne volontiers de poussées ictériques; enfin la forme splé-nique évoque le syndrome de Banti, avec hypertension portale.

Les troubles bíochímíques de la D.H.-L. comportent essentiellement: une hyperamíno-acíduríe massive ( $1 \mathrm{à} 3 \mathrm{~g} / 24 \mathrm{~h}$ ) pouvant atteindre jusqu'aux $15 \%$ (au lieu de 1 à $3 \%$ ) de Tazote urinaire total. Cette perturbation ne s'accompagne pas d'une elevation de l'azote $\alpha$-aminé du sang; elle est indépendante de la gravité de I'atteinte hépatique, et provient d'un défaut de réabsorption tubulaire au niveau du rein.

une surcharge tissulaíre en cuivre. Ce metal circule avant tout (90 à 95\%) lie à une globuline, la caeruloplasmine; dans la D.H-L., cette globuline serait déficiente, et la perturbation fondamentale de la 